VoL. $62(2000) \quad$ [57-66]

\title{
DIRECT SUMS OF INDECOMPOSABLE INJECTIVE MODULES
}

\author{
Sang Cheol Lee and Dong Soo Lee
}

This paper proves that every direct summand $N$ of a direct sum of indecomposable injective submodules of a module is the sum of a direct sum of indecomposable injective submodules and a sum of indecomposable injective submodules of $Z_{2}(N)$.

In this paper, we shall always assume that every ring $R$ has an identity element. By a module, we shall always mean a unitary left $R$-module.

LEMmA 1 . Let $M$ be an $R$-module and suppose that $\left\{M_{i}\right\}_{i \in I}$ and $\left\{N_{i}\right\}_{i \in I}$ are families of submodules of $M$ such that $\sum_{i \in I} \oplus M_{i}=\sum_{i \in I} N_{i}$ in $M$. Suppose further that $M_{i} \supseteq N_{i}$ for each $i \in I$. Then $M_{i}=N_{i}$ for each $i \in I$. tion.

Proof: If $M_{j} \supset N_{j}$ for some $j \in I$, then $\sum_{i \in I} \oplus M_{i} \supset \sum_{i \in I} \oplus N_{i}=\sum_{i \in I} N_{i}$, contradic-

Recall that the singular submodule $Z(M)$ of an $R$-module $M$ is defined by

$$
Z(M)=\left\{m \in M \mid \operatorname{ann}_{R}(m) \text { is essential in } R\right\}
$$

Recall further that the module $M$ is called singular if $M=Z(M)$, and nonsingular if $Z(M)=0$.

It is fairly well known that for any prime $p$ in the ring $\mathbb{Z}$ of integers, the $\mathbb{Z}$-module $G=\mathbb{Z}\left(p^{\infty}\right) \oplus \mathbb{Z}\left(p^{\infty}\right)$ has the property that not every submodule has a unique injective envelope in $G$. It is well-known that if there is an injective envelope of a submodule, within a given nonsingular module, then it is unique. (In fact, this follows from [3, Propositions 4.9, 3.28(b), 3.26] and [2, Lemma 2.1].) This can be further generalised as follows:

LEMMA 2. If there is an injective envelope $E(N)$ of a submodule $N$, within a given $R$-module $M$, then for every injective envelope $E^{\prime}(N)$ of $N$, within $M$,

$$
E^{\prime}(N)+Z(M)=E(N)+Z(M)
$$

Received 23rd September, 1999

The authors wish to acknowledge the financial support of the Korea Research Foundation in the year 1998, Project No. 1998-015-D00010.

Copyright Clearance Centre, Inc. Serial-fee code: 0004-9727/00 \$A2.00+0.00. 
Proof: It is well-known [2, Proposition 1.11] or [3, Proposition 4.9] that there exists an isomorphism $f$ from $E(N)$ onto $E^{\prime}(N)$ such that $\left.f\right|_{N}=i d_{N}$. To show that $E(N)+Z(M)=E^{\prime}(N)+Z(M)$ in $M$, it suffices to prove that for every $e \in E(N) \backslash N$, $\operatorname{ann}_{R}(e-f(e))$ is essential in $R$.

Let $\mathfrak{I}$ be a non-zero left ideal in $R$. Since $N$ is essential in $E(N)$, it follows that $E(N) / N$ is singular. This implies that $\operatorname{ann}_{R}(e+N)$ is essential in $R$, so that $\mathfrak{J} \cap \operatorname{ann}_{R}(e+N) \neq 0$. Take a non-zero element $i$ in $\mathfrak{I} \cap \operatorname{ann}_{R}(e+N)$. Then $i e \in N$.

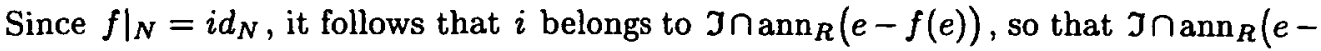
$f(e)) \neq 0$. Hence, $\operatorname{ann}_{R}(e-f(e))$ is essential in $R$, as required.

If $M$ is a nonsingular $R$-module which is a sum of indecomposable injective submodules, then it is a direct sum of indecomposable injective submodules. The generalisation of this will be discussed.

Proposition 3. Let $M$ be an $R$-module which can be expressed in the form $M=\sum_{i \in I} M_{i}+Z(M)$, where the $M_{i}$ 's are indecomposable injective submodules of $M$. Then there exists a subset $J$ of $I$ such that $M=\sum_{j \in J} \oplus M_{j}+Z(M)$.

Proof: Consider the family $\left\{M_{i}\right\}_{i \in I}$. By Zorn's lemma, the collection of subfamilies $\left\{M_{k}\right\}_{k \in K}(K \subseteq I)$ of the family $\left\{M_{i}\right\}_{i \in I}$ such that the sum $\sum_{k \in K} M_{k}$ is direct, has a maximal member, say $\left\{M_{j}\right\}_{j \in J}(J \subseteq I)$. Let $C=\sum_{j \in J} \oplus M_{j}+Z(M)$. In order to show the proposition, it is sufficient to prove that $M=C$.

Suppose that $M \neq C$. Then there exists $i \in I$ such that $M_{i}$ is not contained in $C$. By the maximality of $\left\{M_{j}\right\}_{j \in J}$, we have $M_{i} \cap \sum_{j \in J} \oplus M_{j} \neq 0$. We can now pick out a finite collection $\left\{M_{j_{1}}, M_{j_{2}}, \ldots, M_{j_{r}}\right\}$ of members of $\left\{M_{j}\right\}_{j \in J}$ such that

$$
M_{i} \cap\left(M_{j_{1}} \oplus \cdots \oplus M_{j_{r}}\right) \neq 0 .
$$

Since $M_{j_{1}} \oplus \cdots \oplus M_{j_{r}}$ is injective, $M_{i} \cap\left(M_{j_{1}} \oplus \cdots \oplus M_{j_{r}}\right)$ has an injective envelope which is a submodule of $M_{j_{1}} \oplus \cdots \oplus M_{j_{r}}$ (see [5, Proposition 2.22]). Further, $M_{i}$ is an injective envelope for $M_{i} \cap\left(M_{j_{1}} \oplus \cdots \oplus M_{j_{r}}\right)$. Therefore, according to Lemma 2,

$$
M_{i} \subseteq M_{i}+Z(M) \subseteq M_{j_{1}} \oplus \cdots \oplus M_{j_{r}}+Z(M) \subseteq C .
$$

This is a contradiction. Hence, $M=C$.

Let $A$ be any indecomposable injective $R$-module and let $B$ be any non-zero submodule of $A$. Then $A$ is an injective envelope for $B$. In particular, $B$ is essential in $A$. Hence, the residue class $R$-module $A / B$ is singular. This shows that the homomorphic image of an indecomposable injective $R$-module $A$ is either an indecomposable injective $R$-module (which is isomorphic to $A$ ) or a singular $R$-module. 
Every homomorphic image $N$ of a sum of indecomposable injective submodules of an $R$-module is the sum of a direct sum of indecomposable injective submodules which are not singular and $Z(N)$. More precisely, we proceed to the following:

THEOREM 4 . Let $M$ and $N$ be $R$-modules and let $\left\{M_{i}\right\}_{i \in I}$ be a family of indecomposable injective submodules of $M$. Then for every epimorphism $f: \sum_{i \in I} M_{i} \rightarrow$ $N$, there is a subset $J$ of $I$ such that

$$
N=\left(\sum_{j \in J} \oplus f\left(M_{j}\right)\right)+Z(N)
$$

in which each $f\left(M_{j}\right)$ is indecomposable injective but not singular.

Proof: Let $\left\{M_{i}\right\}_{i \in I}$ be a family of submodules of an $R$-module $M$. Let $N_{i}=$ $f\left(M_{i}\right)$ for each $i \in I$. Then $N=\sum_{i \in I} N_{i}$. Hence,

$$
N=\left(\sum_{i \in I} N_{i}\right)+Z(N) .
$$

Now, assume that each $M_{i}$ is indecomposable injective. Then, since $N_{i}$ is the homomorphic image of $M_{i}$, it follows from the argument immediately following the proof of Proposition 3 that each $N_{i}$ is either indecomposable injective or singular. It may be assumed that every singular submodule of $N$ is contained in $Z(N)$. Thus, we may assume that each $N_{i}$ is indecomposable injective but not singular.

Therefore, by Proposition 3, there is a subset $J$ of $I$ such that

$$
N=\left(\sum_{j \in J} \oplus N_{j}\right)+Z(N)
$$

and each $N_{j}$ is indecomposable injective but not singular, as required.

Theorem 4 provides us with the natural generalisation of a theorem of Harada [4, (8.2.7)].

If $R$ is a nonsingular ring, then the factor $R$-module $M / Z(M)$ is nonsingular (see [3, Proposition 3.29].) However, we cannot say in general that the factor $R$-module $M / Z(M)$ is nonsingular.

Let $R$ be a ring and let $M$ be an $R$-module. Since $Z(M / Z(M))$ is a submodule of the factor $R$-module $M / Z(M)$, it follows from the one-to-one correspondence theorem for modules that there exists a unique submodule $G$ of $M$ containing $Z(M)$ such that

$$
G / Z(M)=Z(M / Z(M))
$$


Then $G$ is called the Goldie torsion submodule (or second singular submodule) of $M$ and is denoted by $Z_{2}(M)$ (see [7]).

We should mention three well known facts. The first is that

$$
\frac{M / Z(M)}{Z_{2}(M) / Z(M)} \cong M / Z_{2}(M)
$$

The second is that $Z_{2}(M) / Z(M)$ is the singular submodule of the factor $R$-module $M / Z(M)$. The third is that the factor $R$-module $M / Z_{2}(M)$ is nonsingular. Clearly, $Z_{2}\left(Z_{2}(M)\right)=Z_{2}(M)$.

THEOREM 5. Let $M$ and $N$ be $R$-modules and let $\left\{M_{i}\right\}_{i \in I}$ be a family of indecomposable injective submodules of $M$. Then for every epimorphism $f: \sum_{i \in I} M_{i} \rightarrow$ $N$, there are subsets $K, J$ of $I$ with $K \subseteq J$ such that

$$
N=\left(\sum_{k \in K} \oplus f\left(M_{k}\right)\right) \oplus Z_{2}(N)
$$

where each $f\left(M_{k}\right)$ is indecomposable injective and nonsingular;

$$
Z_{2}(N)=\left(\sum_{j \in J \backslash K} \oplus f\left(M_{j}\right)\right)+Z(N)
$$

where each $f\left(M_{j}\right)$ is indecomposable injective, not singular but $Z_{2}\left(f\left(M_{j}\right)\right) \neq 0$.

PROOF: By Theorem 4, there is a subset $J$ of $I$ such that

$$
N=\left(\sum_{j \in J} \oplus N_{j}\right)+Z(N)
$$

where $N_{j}=f\left(M_{j}\right)$ and $N_{j}$ is indecomposable injective but not singular. Then since $Z_{2}(N)$ contains $Z(N)$, it follows that

$$
N=N+Z_{2}(N)=\left(\sum_{j \in J} \oplus N_{j}\right)+Z(N)+Z_{2}(N)=\left(\sum_{j \in J} \oplus N_{j}\right)+Z_{2}(N)
$$

Let $K$ be the set of all $j \in J$ with $Z_{2}\left(N_{j}\right)=0$. Then

$$
N=\left(\sum_{k \in K} \oplus N_{k}\right) \oplus\left(\sum_{j \in J \backslash K} \oplus N_{j}\right)+Z_{2}(N) .
$$

Let $j$ be any element of $J \backslash K$. Since $N_{j}$ is indecomposable injective and $Z_{2}\left(N_{j}\right) \neq 0$, $N_{j} / Z_{2}\left(N_{j}\right)$ is both singular and nonsingular. This implies that $N_{j}=Z_{2}\left(N_{j}\right)$. Hence,

$$
\sum_{j \in J \backslash K} \oplus N_{j}=\sum_{j \in J \backslash K} \oplus Z_{2}\left(N_{j}\right) \subseteq Z_{2}(N) .
$$


Further, by making a direct computation, we can see that

$$
\left(\sum_{k \in K} \oplus N_{k}\right) \cap Z_{2}(N) \subseteq \sum_{k \in K} \oplus Z_{2}\left(N_{k}\right)=0 .
$$

Therefore, it follows from (2) that

$$
N=\left(\sum_{k \in K} \oplus N_{k}\right) \oplus Z_{2}(N) .
$$

Here, notice that each $N_{k}$ is nonsingular, because $N / Z_{2}(N)$ is nonsingular so that $\sum_{k \in K} \oplus N_{k}$ is nonsingular.

From (1) and (3), we have

$$
\left(\sum_{k \in K} \oplus N_{k}\right) \oplus Z_{2}(N)=\left(\sum_{k \in K} \oplus N_{k}\right) \oplus\left(\sum_{j \in J \backslash K} \oplus N_{j}\right)+Z(N) .
$$

Therefore, by Lemma 1 or by the modularity, we get

$$
Z_{2}(N)=\left(\sum_{j \in J \backslash K} \oplus N_{j}\right)+Z(N)
$$

The remainder of the proof is obvious.

COROLlary 6. Let $M$ be an $R$-module which is a direct sum of indecomposable injective submodules. Then for every direct summand $N$ of $M, N / Z_{2}(N)$ is a direct sum of indecomposable injective submodules.

Theorem 5 says that if $M$ is a module which is a direct sum of indecomposable injective submodules, then every direct summand $N$ of $M$ is a direct sum of a direct sum of indecomposable injective submodules and $Z_{2}(N)$. This implies that $Z_{2}(N)$ is a direct summand of a direct sum of indecomposable injective modules.

We turn our attention to the decomposition of $Z_{2}(N)$ into indecomposable injective submodules when $Z_{2}(N) \neq 0$. More generally, we proceed to the following:

Lemma 7. Let $X, Y, Z$ be submodules of an $R$-module such that $X \oplus Y=$ $X \oplus Z$. Then there exists an isomorphism $\varphi$ from $Y$ onto $Z$ such that for every submodule $B$ of $Y$ and for every submodule $C$ of $Z, \varphi(B) \cap C=(X \oplus B) \cap C$.

Proof: Let $\pi: X \oplus Z \rightarrow Z$ be the canonical projection. Then for any submodule $B^{\prime}$ of $X \oplus Z, \pi\left(B^{\prime}\right)=\left(X+B^{\prime}\right) \cap Z$. If $X \oplus Y=X \oplus Z$, then the composite map $\varphi: Y \stackrel{\text { inc }}{\longrightarrow} X \oplus Y=X \oplus Z \stackrel{\pi}{\longrightarrow} Z$ is an isomorphism. Now, let $B$ be any submodule of $Y$ and let $C$ be any submodule of $Z$. Then

$$
\varphi(B) \cap C=\pi(B) \cap C=(X \oplus B) \cap Z \cap C=(X \oplus B) \cap C,
$$


as required.

Compare the following theorem with Azumaya's Decomposition Theorem [1, Theorem 12.6].

TheOREM 8. Let $M$ be an $R$-module which is a direct sum of indecomposable injective submodules $M_{i}, i \in I$. Then every non-zero direct summand of $M$ has an indecomposable injective direct summand isomorphic to one of the $M_{i}$.

Proof: Let $\left\{M_{i}\right\}_{i \in I}$ be a family of indecomposable injective submodules of an $R$-module $M$ such that $M=\sum_{i \in I} \oplus M_{i}$. Let $N$ be a direct summand of $M$. Then there is a submodule $N^{\prime}$ of $M$ such that $M=N \oplus N^{\prime}$. If $N$ is non-zero, then there exists a finite subset $J$ of $I$ such that $\left(\sum_{j \in J} \oplus M_{j}\right) \cap N \neq 0$. Consider the family $\mathcal{F}$ of all finite subsets $F$ of $I$ such that $\left(\sum_{f \in F} \oplus M_{f}\right) \cap N \neq 0$, and consider the set $S=\{|F| \mid F \in \mathcal{F}\}$, where $|F|$ denotes the number of elements of $F$. Then $S$ is a non-empty subset of the set $\mathbb{N}$ of natural numbers. By the well-ordering property of integers, $S$ has the least element $l$. Then $l=\left|F_{*}\right|$ for some finite subset $F_{*}$ of $I$ with $\left(\sum_{f \in F_{*}} \oplus M_{f}\right) \cap N \neq 0$. Write $F_{*}=\left\{i_{1}, \ldots, i_{l}\right\}$.

Assume that $l=1$. Then $\left(M_{i_{1}} \cap N\right) \cap\left(M_{i_{1}} \cap N^{\prime}\right)=0, M_{i_{1}} \cap N \neq 0$, and $M_{i_{1}}$ is indecomposable injective. So, $M_{i_{1}} \cap N^{\prime}=0$. Hence, by [6, Lemma 2.10], $M_{i_{1}} \oplus N^{\prime}$ is a direct summand of $M$. In fact, there exists a submodule $N_{1}$ of $N$ such that $M=M_{i_{1}} \oplus N^{\prime} \oplus N_{1}$, that is, $N^{\prime} \oplus M_{i_{1}} \oplus N_{1}=N^{\prime} \oplus N$. By Lemma 7 , there exists an isomorphism $\varphi$ from $M_{i_{1}} \oplus N_{1}$ onto $N$. Hence $\varphi\left(M_{i_{1}}\right) \oplus \varphi\left(N_{1}\right)=\varphi\left(M_{i_{1}} \oplus N_{1}\right)=N$ so that $N$ has an indecomposable injective direct summand $\varphi\left(M_{i_{1}}\right)$ which is isomorphic to $M_{i_{l}}$.

Assume now that $l>1$. Then by the minimality of $l$, we must have $\left(M_{i_{1}} \oplus \cdots \oplus M_{i_{l-1}}\right) \cap N=0$. According to [6, Lemma 2.10], $M_{i_{1}} \oplus \cdots \oplus M_{i_{l-1}} \oplus N$ is a direct summand of $M$. In fact, there exists a submodule $N_{1}^{\prime}$ of $N^{\prime}$ such that $M=M_{i_{1}} \oplus \cdots \oplus M_{i_{l-1}} \oplus N \oplus N_{1}^{\prime}$. Write $M$ as follows:

$$
M=M_{i_{1}} \oplus \cdots \oplus M_{i_{l-1}} \oplus \sum_{i \in I \backslash\left\{i_{1}, \ldots, i_{l-1}\right\}} \oplus M_{i}
$$

By Lemma 7, there exists an isomorphism $\varphi$ from $\sum_{i \in I \backslash\left\{i_{1}, \ldots, i_{l-1}\right\}} \oplus M_{i}$ onto $N \oplus N_{1}^{\prime}$ such that $\varphi\left(M_{i_{l}}\right) \cap N=\left(M_{i_{1}} \oplus \cdots \oplus M_{i_{l-1}} \oplus M_{i_{l}}\right) \cap N$. Note that

$$
\left(\varphi\left(M_{i_{l}}\right) \cap N\right) \cap\left(\varphi\left(M_{i_{l}}\right) \cap N_{1}^{\prime}\right)=0
$$


According to the construction of $l$,

$$
\varphi\left(M_{i_{l}}\right) \cap N=\left(M_{i_{1}} \oplus \cdots \oplus M_{i_{l}}\right) \cap N=\left(\sum_{f \in F_{*}} \oplus M_{f}\right) \cap N \neq 0 .
$$

$M_{i_{l}} \cong \varphi\left(M_{i_{l}}\right)$ implies that $\varphi\left(M_{i_{l}}\right)$ is indecomposable injective. Hence, as in the case $l=1$, we can prove that $N$ has an indecomposable injective direct summand which is isomorphic to $\varphi\left(M_{i_{l}}\right)$ and hence to $M_{i_{l}}$.

REMARK. The word "isomorphic" in Theorem 8 cannot be replaced by the word "equal". An example of this is given in the following example.

EXAMPLE 9. Let $R=\mathbb{Z}$ be the ring of integers and let $\mathbb{N}$ be the set of non-negative integers. Let $V$ be a vector space over the field $\mathbb{Z} / 2 \mathbb{Z}$ with a countable basis $v_{i}, i \in \mathbb{N}$. Let $M$ be the injective envelope of the $\mathbb{Z}$-module $V$. Then $M=\sum_{i \in \mathbb{N}} \oplus M_{i}$, where each $M_{i}$ is an indecomposable injective $\mathbb{Z}$-module whose socle is the submodule with two elements $\mathbb{Z} v_{i}$. Let $N$ be an injective envelope in $M$ of the submodule generated by the countable set $v_{i}-v_{i+1}, \quad i \in \mathbb{N}$, and $N^{\prime}$ be an injective envelope in $M$ of the submodule generated by $v_{0}$. Clearly $M=N \oplus N^{\prime}$. Note that $N=Z_{2}(N) \neq 0$. Then

$$
K=\left\{i \in \mathbb{N} \mid Z_{2}\left(M_{i}\right) \neq 0\right\}=\mathbb{N} .
$$

Now $N=Z_{2}(N) \neq 0$ has an indecomposable injective direct summand $N_{1}$ which is isomorphic to $M_{k_{1}}$ for some $k_{1} \in \mathbb{N}$. For instance, take as $N_{1}$ the injective envelope in $M$ of the submodule generated by $v_{0}-v_{1}$. This $N_{1}$ is isomorphic to $M_{k_{1}}$ for any $k_{1} \in \mathbb{N}$. Let $N_{1}^{\prime}$ be a submodule of $N=Z_{2}(N)$ such that

$$
N=Z_{2}(N)=N_{1} \oplus N_{1}^{\prime} .
$$

For instance, take as $N_{1}^{\prime}$ the injective envelope in $M$ of the submodule generated by the countable set $v_{i}-v_{i+1}, i \geq 1$. From this we cannot deduce that

$$
N=Z_{2}(N)=M_{k_{1}} \oplus N_{1}^{\prime} .
$$

Otherwise, the elements $v_{k_{1}}$ in the socle of $M_{k_{1}}$ would belong to $N$. But this would imply that all the elements $v_{i}$ are in $N$, that is, $N=M$, contradiction.

To investigate the Goldie torsion submodule of an indecomposable injective module, we need to introduce the notion of a module with $\left(C_{11}\right)$.

Let $M$ be an $R$-module and let $N$ be a submodule of $M$. By Zorn's lemma, the collection of submodules $L$ of $M$ such that $N \cap L=0$ has a maximal member. A complement of $N$ in $M$ is a submodule $K$ of $M$ maximal with respect to the property 
$N \cap K=0$. A submodule $K$ of $M$ is called a complement in $M$ if there exists a submodule $N$ of $M$ such that $K$ is a complement of $N$ in $M$. A module $M$ is a $C S$-module or satisfies $\left(C_{1}\right)$ if every complement in $M$ is a direct summand of $M$. A module $M$ is said to satisfy $\left(C_{11}\right)$ if every submodule of $M$ has a complement in $M$ which is a direct summand of $M$.

If an $R$-module $M$ satisfies $\left(C_{1}\right)$, then it satisfies $\left(C_{11}\right)$. For example, every injective $R$-module satisfies $\left(C_{1}\right)$ and hence it satisfies $\left(C_{11}\right)$. In fact, let $M$ be an injective $R$-module and let $K$ be any complement in $M$. Then there is a submodule $N$ of $M$ such that $K$ is a complement of $N$ in $M$. Since $M$ is injective and $N \oplus K$ is a submodule of $M, N \oplus K$ has an injective envelope $E(N \oplus K)$ in $M$. Note that $E(N \oplus K)=E(N) \oplus E(K)$. According to [5, Proposition 2.15], $E(N) \oplus E(K)$ is a direct summand of $M$. Let $P$ be a submodule of $M$ such that

$$
M=E(N) \oplus E(K) \oplus P .
$$

Then $N \cap(E(K) \oplus P)=0$ and $K \subseteq E(K) \oplus P$. By the maximality of $K, E(K) \oplus P=$ $K$. Hence $E(K) \subseteq K$ and $P \subseteq K$, so $K=E(K)$ and $P=P \cap K=0$. Thus, $M=E(N) \oplus K$. This shows that $K$ is a direct summand of $M$. Therefore, $M$ satisfies $\left(C_{1}\right)$ and hence it satisfies $\left(C_{11}\right)$.

Proposition 10. Let $M$ be an $R$-module. If $M$ is indecomposable injective, then either $Z_{2}(M)=0$ or $Z_{2}(M)=M$.

Proof: Assume that $M$ is indecomposable injective. Then since $M$ is injective, $M$ satisfies $\left(C_{11}\right), Z_{2}(M)$ is a direct summand of $M$ (see [6, Theorem 2.7].) Since $M$ is indecomposable, either $Z_{2}(M)=0$ or $Z_{2}(M)=M$.

LEMmA 11. Let $M$ be a direct sum of indecomposable injective submodules. Then every direct summand of $M$ is the sum of all its indecomposable injective submodules.

ProOF: Let $M=\sum_{i \in I} \oplus M_{i}$ be a direct sum of indecomposable injective submodules $M_{i}$. Let $N$ be any direct summand of $M$. Let $S$ be the sum of all indecomposable injective submodules of $N$. The goal is to show that $S=N$.

Suppose on the contrary that $S \neq N$. Then we can take an element $x \in N \backslash S$. There is a finite set $\left\{i_{1}, \ldots, i_{m}\right\} \subseteq I$ with $x \in M_{i_{1}} \oplus \cdots \oplus M_{i_{m}}$. Set $A=M_{i_{1}} \oplus \cdots \oplus M_{i_{m}}$. By [1, Lemma 26.4] there are direct summands $P$ of $N$ and $P^{\prime}$ of $N^{\prime}$ such that

$$
M=A \oplus P \oplus P^{\prime}
$$

Let

$$
H=N \cap\left(A \oplus P^{\prime}\right)
$$


Then $x \in N \cap A \subseteq H$, and by modularity

$$
N=N \cap M=N \cap\left(\left(A \oplus P^{\prime}\right) \oplus P\right)=H \oplus P .
$$

Since $P^{\prime}$ is a direct summand of $N^{\prime}$, there is a submodule $P^{\prime \prime}$ of $N^{\prime}$ with $N^{\prime}=P^{\prime} \oplus P^{\prime \prime}$. Thus,

$$
\begin{aligned}
A & \cong M /\left(P \oplus P^{\prime}\right) \\
& =\left(N \oplus N^{\prime}\right) /\left(P \oplus P^{\prime}\right) \\
& =\left(H \oplus P \oplus P^{\prime} \oplus P^{\prime \prime}\right) /\left(P \oplus P^{\prime}\right) \\
& \cong H \oplus P^{\prime \prime} .
\end{aligned}
$$

But, by [1, Corollary 12.7] the decomposition $A=M_{i_{1}} \oplus \cdots \oplus M_{i_{m}}$ complements direct summands. Hence, in particular, $H$ is isomorphic to $\bigoplus_{k \in F} M_{k}$ for some finite subset $F \subseteq I$, so that $H$ is a direct sum of indecomposable injective submodules of $N$. Therefore, we get $x \in H \subseteq S$, contradiction.

Recently, study of direct sum decompositions into indecomposable injective modules has been done in [8].

THEOREM 12. Let $M$ be an $R$-module which is a direct sum of indecomposable injective submodules. Then every direct summand $N$ of $M$ is the sum of a direct sum of indecomposable injective submodules and a sum of indecomposable injective submodules of $Z_{2}(N)$.

Proof: Let $\left\{M_{i}\right\}_{i \in I}$ be a family of indecomposable injective submodules of an $R$-module $M$ such that $M=\sum_{i \in I} \oplus M_{i}$. Let $N$ be a direct summand of $M$. Let $\pi: M \rightarrow N$ be the canonical projection. Then by Theorem 5 , there is a subset $J$ of $I$ such that

$$
N=\left(\sum_{j \in J} \oplus \pi\left(M_{j}\right)\right) \oplus Z_{2}(N)
$$

where each $\pi\left(M_{j}\right)$ is indecomposable injective. Let $N^{\prime}$ be a submodule of $\sum_{i \in I} \oplus M_{i}$ such that $\sum_{i \in I} \oplus M_{i}=N \oplus N^{\prime}$. Then

$$
\sum_{i \in I} \oplus Z_{2}\left(M_{i}\right)=Z_{2}(N) \oplus Z_{2}\left(N^{\prime}\right)
$$

Let

$$
K=\left\{i \in I \mid Z_{2}\left(M_{i}\right) \neq 0\right\}
$$


Then by Proposition 10,

$$
\sum_{k \in K} \oplus M_{k}=Z_{2}(N) \oplus Z_{2}\left(N^{\prime}\right)
$$

Hence, by Lemma 11, $Z_{2}(N)$ is the sum of all its indecomposable injective submodules. By (1), $N$ is a direct sum of a direct sum of indecomposable injective submodules and the sum of all indecomposable injective submodules of $Z_{2}(N)$. Therefore, by Zorn's lemma, $N$ is the sum of a direct sum of indecomposable injective submodules and a sum of indecomposable injective submodules of $Z_{2}(N)$.

\section{REFERENCES}

[1] F.W. Anderson and K.R. Fuller, Rings and categories of modules, (Second Edition) (Springer-Verlag, Berlin, Heidelberg, New york, 1992).

[2] K.R. Goodearl, Ring theory. Nonsingular rings and modules, Pure and Applied Mathematics 33 (Marcel Dekker, Inc., New York, Basel, 1976).

[3] K.R. Goodearl and R.B. Warfield, Jr, An introduction to noncommutative Noetherian rings, London Mathematical Society Student Texts 16 (Cambridge University Press, Cambridge, New York, 1989).

[4] M. Harada, Factor categories with applications to direct decomposition of modules, Notes in Pure and Applied Mathematics 88 (Marcel Dekker, New York, 1983).

[5] D.W. Sharpe and P. Vámos, Injective modules, Cambridge Tracts in Mathematics and Mathematical Physics 62 (Cambridge, London, New York, 1972).

[6] P.F. Smith and A. Tercan, 'Generalizations of CS-Modules', Comm. Algebra 21 (1993), 1809-1847.

[7] B. Stenström, Rings of quotients (Springer-Verlag, Berlin, Heidelberg, New York, 1975).

[8] H.-P. Yu, 'On Krull-Schmidt and a problem of Matlis', Comm. Algebra 24 (1996), 2851-2858.

Department of Mathematics Education

Chonbuk National University

Chonju, Chonbuk 561-756

Korea

e-mail: scl@moak.chonbuk.ac.kr
Department of Mathematics

Chungnam National University

Taejon 305-764

Korea

e-mail: dslee@math.chungnam.ac.kr 